\title{
Assessing mitigation of the risk from extreme wildfires using MODIS hotspot data
}

\author{
$\underline{\text { Rick McRae }}^{a}$ and Jason Sharples ${ }^{b}$ \\ ${ }^{a}$ ACT Emergency Services Agency, Canberra \\ ${ }^{b}$ School of Physical, Environmental and Mathematical Sciences, UNSW Canberra \\ Email: rick.mcrae@act.gov.au
}

\begin{abstract}
A taxing question in wildfire risk management concerns the effectiveness of fuel reduction burning on mitigating the risk to Australia's communities from extreme wildfires. Extreme wildfires account for the majority of wildfire damage over recent years. In part the uncertainty arises from how questions are framed, in part from the lack of effective datasets to conduct definitive analyses. The development of a climatology of satellite-derived fire hotspots over the last thirteen years has permitted new approaches to the problem that yields answers that may provide valid insights into improving community safety.
\end{abstract}

The complexity of the problem necessitated focusing on a study domain. The effectiveness of fuel reduction arises from the inter-annual accumulation of leaf litter in eucalyptus dominated forests. The forests of mainland southeast Australia were selected to meet that criterion in a well understood setting.

Within the domain, included hotspots for each fire year were separated, aggregated and used to generate a $2 \mathrm{~km}$ radius buffer area. This was designed to minimise issues associated with hotspot positional errors and to, in some sense, account for associated milder fire areas that would not trigger the hotspot algorithm. Small scale or low intensity fuel reduction burns are missed by this approach, but they are unlikely to be effective against an extreme wildfire.

If intensive fuel reduction were effective, then there would be an expectation of burnt areas in preceding years affecting, in a measurable way, the extent of an extreme wildfire. To do this, a subset of the buffered area was generated for hotspots associated with extreme wildfires. These were identified based on the expected spatiotemporal clustering of their hotspots and on-going research into these events.

Two hypotheses are explored. Firstly, as is often claimed, fuel reduction burning may be effective. Overlaps of specific sets of hotspots were analysed with respect to this. If this is not the case, this may be due to the small proportion of the domain burning each year, making overlaps unlikely. Secondly, if fuel reduction burning is not effective, this may be due to remoteness and ruggedness. The distributions of extreme wildfires and other fire were compared with respect to these environmental parameters.

By examining the four preceding years it was found that some extreme wildfires were not affected despite appreciable overlap with preceding fuel reduction activity. The main proportion of an extreme wildfire that was recently burnt is estimated at $19 \%$, including the downwind edge. In terms of distance in from the edge of a forested area, it was found that fuel reduction occurred mainly towards the edge, while extreme wildfires were relatively much more frequent in the interior. Further, extreme wildfires present greater operational challenges in rugged landscapes. It was found that fuel reduction is more frequent in flat landscapes, while extreme wildfire is most common on rugged landscapes.

The implications of these patterns for mitigation of the impacts of extreme wildfires are discussed. Statistically, most extreme wildfires are unlikely to encounter previously burnt areas, but that this does not imply that the latter prevents the former. Where they do overlap there is little indication of an interaction. Mitigation of the risks arising from extreme wildfires through fuel management, ignition prevention or response arrangements, must take account of the nature of these fires.

Keywords: $\quad$ Extreme wildfires, hotspots, hazard reduction, ruggedness, remoteness 


\section{INTRODUCTION}

The greatest impacts from wildfires tend to be from large conflagrations associated with violent pyroconvective events. These, which will be called extreme wildfires (as defined in McRae \& Sharples, 2011), may produce a fire thunderstorm, or pyrocumulonimbus (pyroCb for short). PyroCbs have attracted global attention since the turn of the century because they have significant atmospheric impacts at a global scale. They are able to inject aerosols into the stratosphere, where they persist and circulate around the globe (Fromm, et al., 2010).

Studies of the effect of fuel reduction burning on extreme wildfires have only received relatively little attention in the literature - the study by Price and Bradstock (2012) is an exception. Other studies have considered data sets that may include extreme wildfires (e.g. Price et al., 2012), but the effects of prior burning on extreme wildfires in such studies are confounded by its effect on smaller fires. No study has explicitly addressed the effects of fuel reduction burning on the specific sub-class of extreme wildfires. Studies of extreme wildfires have enhanced understanding of their drivers but have not found an explicit role for fuel load in their development (e.g. McRae et al., 2015). The same pyroCb dynamics have been observed in a range of fuel types. The apparent potential for a reduced dependence on fuel load raises serious questions about methods for reducing the risk from these fires. This study explores that issue.

At present, the primary tool for mitigating wildfire risk in Australia is through prescribed fuel (or hazard) reduction burning (HRB) (AFAC, 2015). This is founded on the role of fuel load in the principal fire danger and fire behaviour models used. If fuel load is kept low, the modelled fire rate of spread and intensity drop to levels that may permit effective fire suppression or prevention of damage to assets. Large numbers of HRBs are lit every year (here meaning a fire year from July to June), and these span a wide range of sizes and intensities. As they are lit by a wide range of organisations across a number of jurisdictions, collating records on them was outside the scope of this study.

Since 2003, a consistent global climatology of satellite hotspots from TERRA and AQUA MODIS sensors has been developed. This is now adequate to use as a tool for addressing the relationship between extreme wildfires and HRBs. Satellite hotspots are produced by an algorithm that assesses whether a radiative anomaly exists in the data from any grid-cell in an image (see, for example, Giglio, et al. (2003) and Giglio (2013)). The majority of these are due to fire, although some are due to anomalous surface heating and industrial plant. They indicate that values averaged across an entire pixel (nominally $250 \mathrm{~m}$ on a side) are hot. Small HRBs will fail to be identified by the algorithm, as might burns whose emissions fall into multiple pixels or are obscured by cloud.

The aim of this paper is thus to generate spatial polygons for extreme wildfires, other fires and their environment and to explore their intersections. Any clear patterns are explored to see if they provide any guidance for followup studies into risk mitigation.

\section{DATA \& METHODS}

Given the assumptions that underlie fuel reduction, this study has been limited to a large area of vegetation that accumulates fuel on an interannual basis. The study domain chosen was the forested parts of mainland southeast Australia. The complexities of its fringe made a detailed rendering too difficult to work with, so a set of $0.1^{\circ} \times 0.1^{\circ}$ grid-cells were used to indicate the wholly or largely forested parts of the domain (Figure 1a). We refer to this set of grid cells as the study area and denote it by $A$. Similarly, we will refer to the set of hotspot grid cells corresponding to hazard reduced areas for fire year $j$ as $H_{j}$, and the set of hotspot grid cells corresponding to extreme wildfires in fire year $j$ as $W_{j}$. The extreme wildfires and fire years considered in this study are listed in Table 1. We further partition the set of extreme wildfire grid cells for year $j$ into those corresponding to the left flank, the right flank, the head, the back and the interior, which are denoted $W_{j}^{l}, W_{j}^{r}, W_{j}^{h}, W_{j}^{b}$, and $W_{j}^{i}$, respectively (see Figure 1f). This partitioning is based on the predominant wind direction during the extreme wildfires evolution.

Hotspot data were supplied by the Fire Information and Resource Management System (FIRMS) service, provided by NASA and the University of Maryland (NASA, 2015).

An extreme wildfire occurs on a large spatial scale compared to image pixels, and so it is deemed an acceptable assumption that extreme wildfires will be little affected by HRB activity that does not generate a hotspot. Thus the study reported here assesses the relationship between broad hazard reduced areas and extreme wildfires. To simplify this study, for any year the set of hazard reduced areas is defined by all hotspots from the previous 4 years (whether from wildfire or HRB). 
R.H.D. McRae and J.J. Sharples, Assessing mitigation of the risk from extreme wildfires...

The domain was analysed in terms of terrain ruggedness. Rugged terrain has an elevation range ${ }^{1}$ within $1.5 \mathrm{~km}$ of any point of greater than $300 \mathrm{~m}$, using the SRTM DEM data at $200 \mathrm{~m}$ resolution. Flat terrain has an elevated range of less than $150 \mathrm{~m}$, while undulating terrain is in between. These classes are shown in Figure $1 \mathrm{~b}$, and the breakdown of terrain class by area is $19 \%$ flat, 39\% undulating and $43 \%$ rugged. The subset of domain grid cells classed as flat is denoted $T_{1}$, undulating as $T_{2}$ and rugged as $T_{3}$.

a)

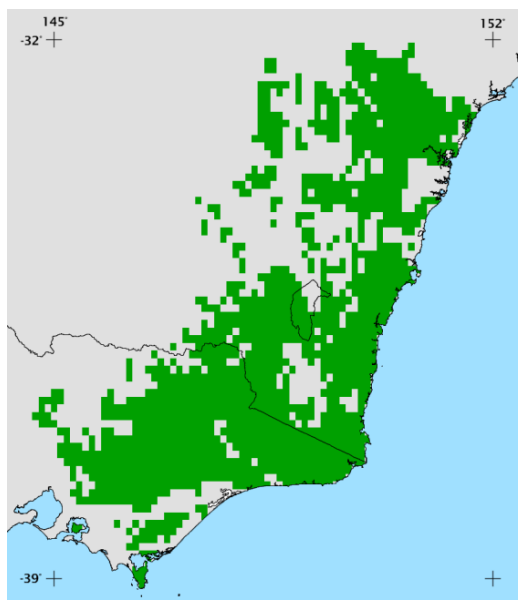

c)

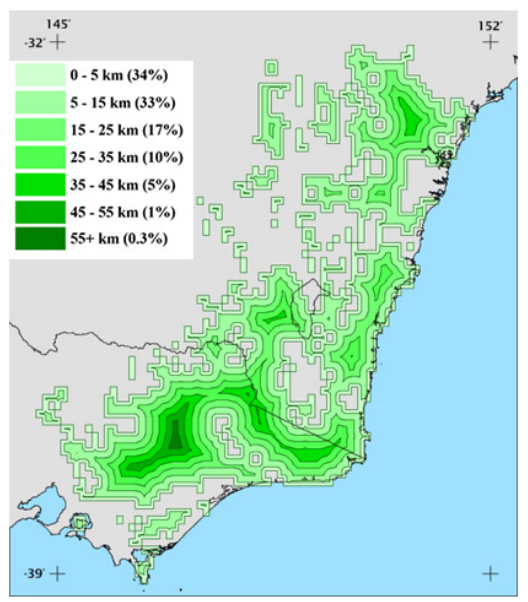

$-32+$

e)

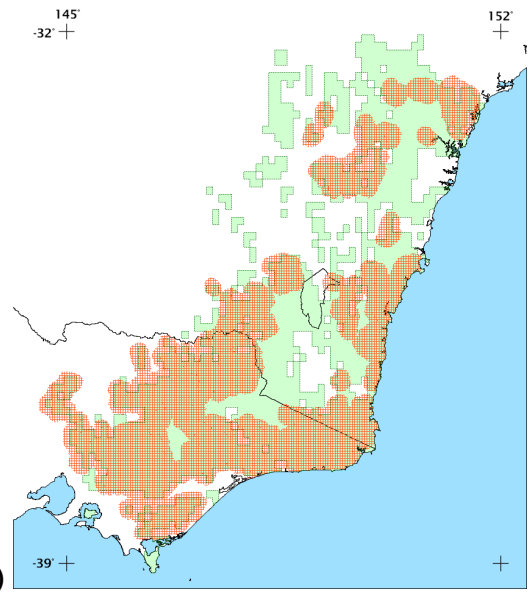

b)

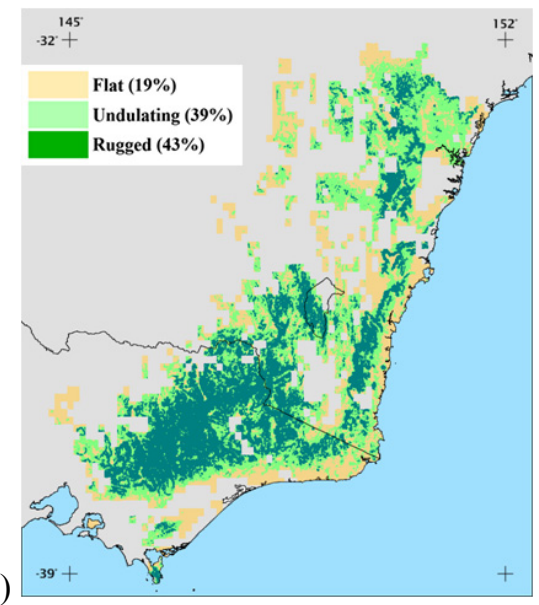

d)
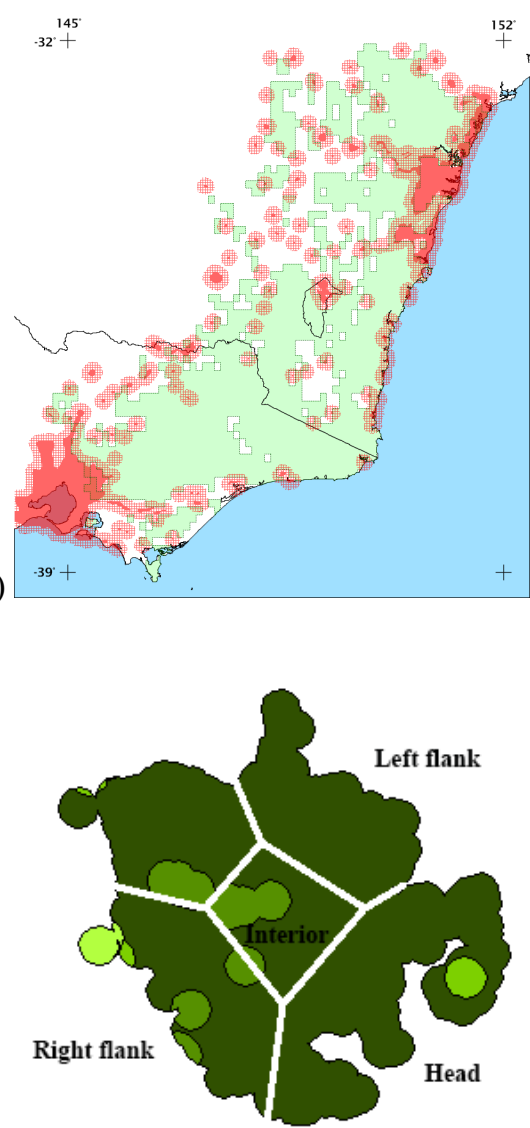

f)

Figure 1. (a) study domain; (b) remoteness classes; (c) ruggedness classes; (d) developed area buffer; (e) forestry area buffer; (f) extreme wildfire stylized zonation, showing also how the extents of the extreme wildfire (dark green) and hazard reduced areas (lighter greens) have been constructed from buffered hotspots.

Additionally, a categorical measure of remoteness was developed, using buffers inwards from the domain edge. The first remoteness class spanned 0 to $5 \mathrm{~km}$, with subsequent remoteness classes defined by moving inwards in

${ }^{1}$ http://www.highfirerisk.com.au/maps/terrain_range.htm, accessed 24 July 2015. 
$10 \mathrm{~km}$ increments. The initial remoteness class was set to $5 \mathrm{~km}$ width to avoid skewing the results due to the convoluted perimeter of the domain. The subset of domain grid cells in remoteness class $n$ is denoted $R_{n}$, with $n$ increasing from 1 (least remote) to 7 (most remote). The classes are shown in Figure 1c.

Where an extreme fire was known to have occurred, its hotspots were aggregated and given an additional $2 \mathrm{~km}$ buffer to provide a consistent rendering of the areas of most intense fire activity. As HRBs are concentrated around key assets at risk, two asset types were established and given $10 \mathrm{~km}$ buffers. These were developed areas (Figure 1d) and forestry areas (Figure 1e). The set of developed areas is denoted $D$ and the set of forestry areas is denoted $F$.

The following notation is used to express the degree of coincidence of two spatial polygons $A$ and $B$ :

$$
\|B\|_{A}=\frac{|B \cap A|}{|A|} .
$$

This quantity is interpreted as the size of polygon $B$ modulo the polygon $A$; and so for example, $\left\|H_{j}\right\|_{W_{j}}$ denotes the number of hazard reduced grid cells for fire year $j$ that coincide with wildfire affected grid cells in fire year $j$, expressed as a proportion of the total number of wildfire affected grid cells. Such terms are expressed as percentages in what follows.

In this study five fire years are considered. The following notation is used to denote the average overlap between polygons over the five fire years:

$$
\mu_{W}(H)=\frac{1}{5} \sum_{j=1}^{5}\left\|H_{j}\right\|_{W_{j}} .
$$

\section{RESULTS}

\subsection{Hazard reduced areas}

The mean overlap between extreme wildfires and hazard reduced areas was found to be $\mu_{W}(H)=19 \%$, which implies that when extreme wildfires do occur, they are not burning solely in areas with untreated hazard. They are, to some extent at least, able to burn over fuel reduced areas.

We could also, a priori, expect this to be true because $\|D\|_{H}=5.3 \%$ and $\|D\|_{W}=5.0 \%$ for years with extreme wildfires (about half). This implies that both extreme wildfires and hazard reduced areas are infrequent and so unlikely to overlap strongly. This makes the previous result somewhat surprising, and suggests that extreme wildfires and hazard reduced areas are not spatially independent. Human activity, which is concentrated in areas likely to be treated with HRBs may to some extent be responsible for fire ignitions that become extreme wildfires.

The mean overlap of various potential influences on the spread of a fire were estimated for different parts of the extreme wildfire sets (listed in Table 1) as follows:

$$
\begin{aligned}
& \mu_{W^{l}}(H)=26 \%, \quad \mu_{W^{i}}(H)=13 \%, \quad \mu_{W^{r}}(H)=14 \%, \quad \mu_{W^{h}}(H)=18 \%, \\
& \mu_{W^{h}}\left(T_{3}\right)=19 \%, \quad \mu_{W^{h}}(D)=66 \%, \quad \mu_{W^{h}}(F)=66 \% .
\end{aligned}
$$

If the forward spread of a fire had been halted by hazard reduced areas then we would expect to see a high degree of overlap with hazard reduced areas in the head zone. The value of $\mu_{W^{h}}(H)=18 \%$ suggests there is some truth to this proposition.

However given the value of $\mu_{W^{h}}(H)$ we need to test for a marked increase in hazard reduced areas near the head above that in the other parts of extreme wildfires, especially the interior zone. The value for the interior parts of extreme wildfires is $\mu_{W^{i}}(H)=13 \%$, however it is not clear if this is a significantly lower value. So while this may imply that the headfire abates due to burning into hazard reduced areas, it can also be claimed that extreme wildfires are able to spread through a broadly similar amount of hazard reduction without abating.

Across all of the parts of an extreme wildfire, if hazard reduced areas affected the spread pattern of extreme wildfires, then we would expect to see relatively higher frequencies of hazard reduced areas for some parts (especially the left and right flanks). This is perhaps only seen for the left flank. In other words there is no strong indication of a tendency for parts of an extreme wildfire to halt due to entering hazard reduced areas. The somewhat raised value for the left flank suggests that HRB effort might be less effective in halting the large conflagrations that emerge after a major wind change. Further scrutiny is required. 
R.H.D. McRae and J.J. Sharples, Assessing mitigation of the risk from extreme wildfires...

Table 1. Details of the extreme wildfire events and fire years considered in the study.

\begin{tabular}{|c|c|c|c|c|c|}
\hline \multirow[t]{2}{*}{ Event } & \multicolumn{4}{|c|}{ Hazard reduced area coincidence with fire parts (\%) } & \multirow{2}{*}{$\begin{array}{l}\text { Stopped outside rugged terrain class? } \\
\qquad 1=\text { Yes, } 0=\text { No }\end{array}$} \\
\hline & Left flank & Head & Right flank & Interior & \\
\hline 0203a & na & na & na & na & 1 \\
\hline 0203b & na & na & na & na & 0 \\
\hline 0607a & 80 & 10 & 10 & 5 & 1 \\
\hline 0607b & 0 & 25 & 30 & 10 & 0 \\
\hline 0607c & 0 & 0 & 15 & 0 & 1 \\
\hline 0809a & 80 & 15 & 20 & 70 & 0 \\
\hline 0809b & 50 & 10 & 40 & 50 & 0 \\
\hline 0809c & 80 & 0 & 0 & 30 & 1 \\
\hline 0809d & 5 & 20 & 25 & 30 & 1 \\
\hline 0809e & 50 & 50 & 10 & 50 & 0 \\
\hline $1213 a$ & 0 & 0 & 0 & 0 & 0 \\
\hline $1213 b$ & 0 & 5 & 5 & 15 & 1 \\
\hline 1314a & 5 & 5 & 0 & 0 & 0 \\
\hline $1314 b$ & 5 & 0 & 5 & 5 & 0 \\
\hline $1314 c$ & 5 & 10 & 0 & 0 & 0 \\
\hline $1314 d$ & 15 & 0 & 0 & 0 & 0 \\
\hline $1314 \mathrm{e}$ & 20 & 0 & 30 & 20 & 0 \\
\hline $1314 \mathrm{f}$ & 15 & 40 & 40 & 0 & 1 \\
\hline Mean & 26 & 13 & 14 & 18 & 0.39 \\
\hline
\end{tabular}

\subsection{Remoteness class}

The distribution of hazard reduced areas and extreme wildfires with respect to remoteness is shown in Figure 2. Extreme wildfire is most common in the most remote areas, while hazard reduction is most common in the least remote areas. The inference is that extreme wildfires are less likely to encounter hazard reduced areas in the more remote areas. The ratios of the two are shown in Figure 2: a clear trend is evident, with extreme wildfire occurrence becoming increasingly frequent compared to hazard reduction as the landscape becomes more remote.

Moreover, hazard reduced areas are likely to experience extreme wildfires as a result of their escalation (a blowup event). At this stage of fire development, the dynamics of extreme wildfire propagation, which includes effects such as intense, atypical spread, ember storms, the lack of a well-defined head fire and strong vorticity, poses the greatest challenge to the efficacy of hazard reduction. This raises the notion that HRB effort could be more effective if implemented in greater concentration in remote areas - this way they may have an improved mitigative effect on ignitions before they can escalate into extreme wildfires.

\subsection{Ruggedness class}

The relationship with landscape ruggedness can be explored in a similar way to remoteness. Figure 3 shows the distribution of extreme wildfires and hazard reduction across the different terrain classes. In flat landscapes access is easiest and operations are simpler, while undulating and rugged landscapes provide major challenges to all aspects of fire management. Figure 3, however, indicates that it is in these terrain classes where extreme wildfire is most frequent. Seven out of 18 extreme wildfires stopped forward spread less than $5 \mathrm{~km}$ after leaving rugged landscapes. The notion that ruggedness acts to assist extreme wildfire development is supported. A number of recent studies into the dynamics of extreme wildfire development also support this notion (e.g. McRae et al. (2015) and the references therein).

\subsection{Developed areas}

HRB activity around developed areas might be expected to have a specific impact on extreme wildfires. The distribution of developed area within the extreme wildfires studied is shown in Figure 4. The full range of values is represented, with $44 \%$ of extreme wildfires scoring near $0 \%$, and $22 \%$ near $100 \%$. There is no evident clustering that would indicate a strong effect of developed areas on extreme wildfire.

\subsection{Forestry areas}

HRB activity around forestry areas might also be expected to have a specific impact on extreme wildfires. The distribution of forestry area within the extreme wildfires studied is shown in Figure 5. The full range of values is represented, with $16 \%$ of extreme wildfires scoring near $0 \%$, and $50 \%$ near $100 \%$. Again, no strong effect is indicated. The higher average values for forestry area than for developed area reflects the ability of broad treatment areas within the domain to carry an extreme wildfire. In contrast, the core of developed areas cannot carry these fires, is largely outside of the study area, tends not to be rugged and is (by definition) not remote. 


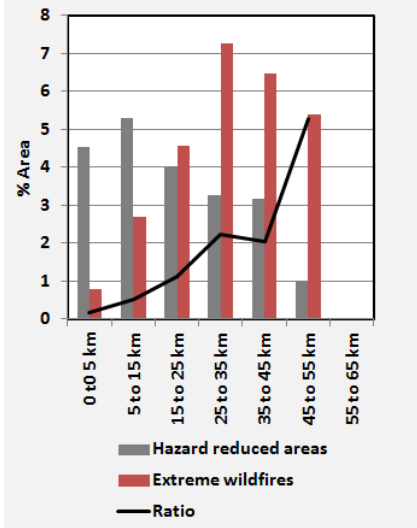

Figure 2. Distribution of hazard reduced areas and extreme wildfires by remoteness class (all years). Also shown is the ratio of extreme wildfires to hazard reduced areas.

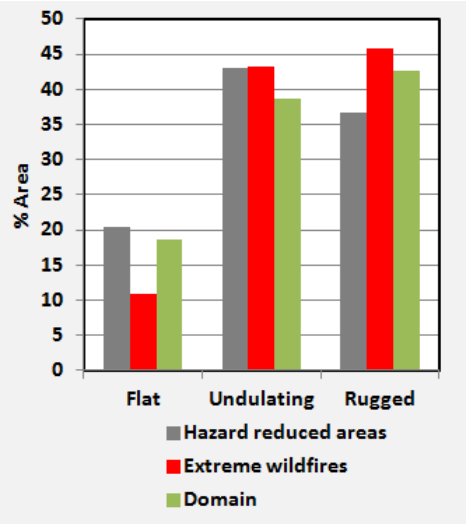

Figure 3. Distribution of extreme wildfires by ruggedness class (all years).

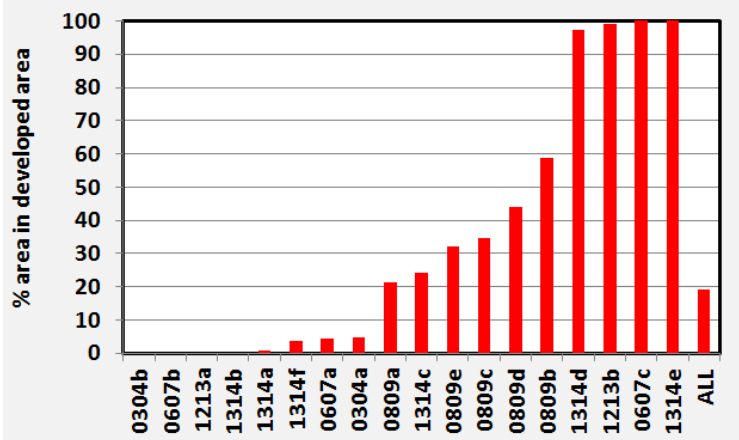

Figure 4. Ranked distribution of developed area between extreme wildfires.

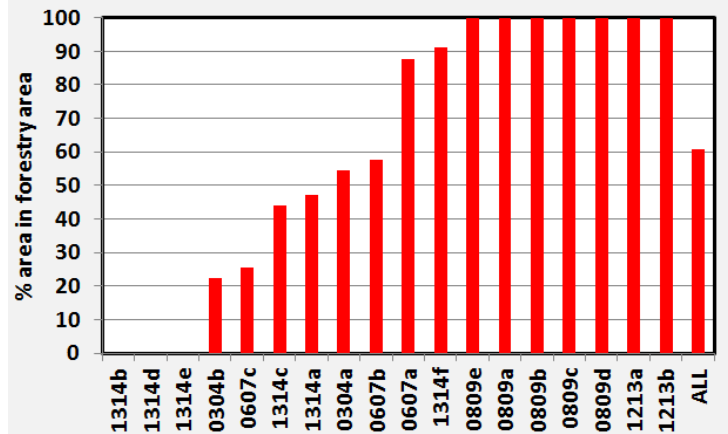

Figure 5. Ranked distribution of forestry area between extreme wildfires.

\section{DISCUSSION}

Extreme wildfires and indeed all forms of satellite detectable fires are shown to cover such a small part of the landscape that their burnt areas do not often overlap or interact. That means that hazard reduction effort will be unlikely to be intensive enough to influence the evolution of extreme wildfires. Significant re-burns do occur, as in the re-burning of the 0304a fire area in the Mount Hotham area (Vic) in the 0607a and 0809e extreme wildfires, or in the high hazard reduced areas frequency in the 0809a Kilmore East wildfire.

Many fires start in non-rugged landscapes or in easily accessible areas and are put out. Some ignitions develop and ultimately encounter conditions conducive to blow-up fire events (as defined in McRae and Sharples, 2013; 2014). These form extreme wildfires. This is more likely to occur in remote or rugged areas where rapid, effective response and suppression will be most difficult. These are also where hazard reduced areas are least likely.

If an extreme wildfire moves out of rugged or remote terrain, or moves towards developed areas or forestry areas, it will encounter more hazard reduced areas. By then the fire will have escalated to a large scale and would exhibit atypical fire dynamics. These aspects combine to make hazard reduced areas less effective, and may produce a level of impact disproportionate to the HRB effort.

It is necessary that future work explores the finding that 7 out of 18 extreme wildfires stopped soon after leaving rugged landscapes. This transition corresponds to changes in access, vegetation, HRB intensity and weatherterrain interactions. It is important to find the independent variables here. As mentioned, fuel load currently has no explicit role in the study of extreme wildfires. It is hoped that this can be investigated with a view to either giving it that role or confirming that the role does not exist.

The analyses above are all based on a rather specific set of treatments of hotspot data and the forested study domain. It is accepted that there are limitations to this approach. However, the methodology has the benefit of being consistent across the domain and between fire years. Indeed, it circumvents issues that might otherwise be expected when combining datasets from a range of jurisdictions or fire management agencies. 
Despite the limitations of the study design, some valid conclusions may be drawn from the study. The hope is that these conclusions will drive follow-on studies that improve on the limitations of the current study and provide greater statistical rigour. It is expected, given the strengths of this study, that new work will refine the conclusions reached, rather than change them. Further study will be facilitated through fire agencies providing new datasets designed for this purpose from future extreme wildfires. Resources such as the new Himawari-8 weather satellite will likely prove valuable in this respect.

What risk mitigation options are suggested by this study? Firstly, a more extensive hazard reduction program that produces a larger coverage of hazard reduced areas might be effective. However, the funding and resourcing of this becomes an issue needing consideration. Secondly, more targeted hazard reduction burning in remote and rugged areas that produce larger hazard reduced areas in those regions might be effective in mitigating the risk from extreme wildfires. Again the costing and resourcing need to be addressed, and the perceptions of lower concentration of effort close to life and property might result in political issues.

There is also an indication that a greater emphasis on ignition prevention may reduce the risk. This risk treatment is limited, however, as it would not affect lightning ignitions. Fire agencies could augment their initial response capabilities, with the aim of preventing newly ignited fires from escalating, though budgetary constraints may limit this risk treatment.

Given the relatively high levels of hazard reduced areas within the 0809a-e extreme wildfires there might be no consistently effective fuel treatment for extreme wildfire-derived risks. Also the observation that extreme wildfires such as 0304a and 0607a only stopped after leaving rugged landscapes, suggests there may be areas in or adjacent to rugged landscapes where the residual risk is elevated.

\section{REFERENCES}

AFAC (2015). Overview of Prescribed Burning in Australasia. Report for the national Burning Project subproject 1. Australasian Fire and Emergency Services Authorities Council Limited. March 2015.

Fromm, M., Lindsey, D.T., Servranckx, R., Yue, G., Trickl,, T., Sica, R., Doucet, P. and Godin-Beekmann, S. (2010). The Untold Story of Pyrocumulonimbus. Bulletin of the American Meteorological Society. September 2010, pp 1193 - 1209.

Giglio, L. (2013). MODIS Collection 5 Active Fire Product User's Guide. Version 2.5. Department of Geographical Sciences, University of Maryland, 61pp.

Giglio, L., Descloitres, J., Justice, C.O. and Kaufman, Y.J. (2003). An Enhanced Contextual Fire Detection Algorithm for MODIS. Remote Sensing and Environment, 87, 273-282.

McRae, R. \& Sharples, J. (2011). A conceptual model for assessing the risk posed by extreme bushfires. Australian Journal of Emergency Management, 26(2), 47-53.

McRae, R.H.D. and Sharples, J.J. (2013). A process model for forecasting conditions conducive to blow-up fire events. Proceedings, 2013 MODSIM Conference, Adelaide.

McRae, R.H.D. and Sharples, J.J. (2014). Forecasting conditions conducive to blow-up fire events. CAWCR Research Letters, 11, 14-19.

McRae, R. H. D., Sharples, J.J. and Fromm, M. (2015). Linking local wildfire dynamics to pyroCb development. Natural Hazards Earth System Sciences, 15, 417-428.

NASA (2015). Fire Information for Resource Management System (FIRMS). (URL: https://earthdata.nasa.gov/earth-observation-data/near-real-time/firms, accessed 24 July 2015.)

Price, O.F. and Bradstock, R.A. (2012). The efficacy of fuel treatment in mitigating property loss during wildfires: Insights from analysis of the severity of the catastrophic fires in 2009 in Victoria, Australia. Journal of Environmental Management 113, 146-157.

Price, O.F., Bradstock, R.A. Keeley, J.E. and Syphard, A.D. (2012). The impact of antecedent fire area on burned area in southern California coastal ecosystems. Journal of Environmental Management 113, 301-307.

Russell-Smith, J. Yates, C.P., Whitehead, P.J., Smith, R., Craig, R., Allan, G.E., Thackway, R., Frakes, I., Cridland, S., Meyer, M.C.P. and Gill, A.M. (2007). Bushfires 'down under': patterns and implications of contemporary Australian landscape burning. International Journal of Wildland Fire, 16, 361-377. 